\title{
Community Pharmacy Experiences and Attitudes towards Diabetes Managment
}

\author{
Ala’ M Yasin, Suhair S Al Tarawneh, Mosheer Kamil. Al. Mogasgas, Samar Younes Al hawary, Sahar \\ Mohammed Jdaitawi and Tareq Mukatash \\ Department of Clinical Pharmacy, Faculty of Pharmacy, Jordan University of Science and Technology, Irbid 962507, Jordan
}

\begin{abstract}
To measure the experiences, knowledge and attitudes of community pharmacists in Jordan towards diabetes and its management. The validated diabetes knowledge survey tool obtained from Simpson et al, 2009 was modified to suit the Jordanian population of community pharmacists. It contains two sections related to the services that pharmacists provided to diabetics patients and attitude toward health care professionals. The random sampling selection method was used and oral interviews using modified questionnaires were administered to measure the attitudes of pharmacists in Jordan towards diabetes. Random selection was achieved via numbering lists of 1,000 community pharmacists with 500 being chosen randomly across the country. In a total of 422 completed survey (84.4\%), more than $60 \%$ of the pharmacists showed that they "sometimes" counsel diabetic patients. In addition, home blood glucose monitoring was promoted by around half of them. Only a small part of them showed that that they checked prescriptions for drug interactions. The most of them showed not to provide patients with information regarding insulin use and storage. The most of them showed that absence of time and lack of continuous education programs are the main barriers limiting community interaction with diabetic patients. Pharmacists should be provided with continuous diabetes education programs to increase and enhance their counseling skills. The community pharmacists should play a greater role toward the well being of diabetic patients.
\end{abstract}

Key words: Community pharmacy, attitudes, diabetes management.

\section{Introduction}

Diabetes mellitus affects approximately 1 in 7 Jordanians [1]. Recently, there has been an increasingly growing role for community pharmacists to help people in prevention and management of diabetes [2].

Since then, a reduction of diabetes risk by more than $50 \%$ has been publicly claimed and large-scale population intervention is being promoted [3]. A Canadian study about the attitudes of pharmacists toward diabetes and its management concluded that pharmacists agree that they should be part of the health-care team for managing diabetes and should be required to have specialized training to provide primary diabetes care [4].

The pharmacist's role in diabetes management is

Corresponding author: Ala' M Yasin, M.sc., research fields: clinical pharmacy and faculty of pharmacy. E-mail: Alaayassien@yahoo.com. defined by generalizing the activities associated with patient identification, screening patients at high risks, assessment for drug-related problems (focusing on barriers to optimal glucose control), patient education, referral to other members of the diabetes management team and ongoing monitoring [5-7]. Different studies have concluded that when pharmacists take part in identifying and managing drug-related problem, there will be consistent improvement in the treatment outcomes [8-10].

In this study, the author aimed to assess the current situation in Jordan with regards to diabetes related pharmacy care and counseling. The latter was achieved through a semi structured questionnaire distributed to a sample of randomly selected community pharmacists in Jordan.

\section{Methods}

The questionnaire: A questionnaire obtained from 
[11]. The questionnaire was used to measure the relationship between diabetes-specific training and pharmacists' activities and attitudes toward diabetes management.

The survey was divided into two sections; the first section contains 22 questions that coverdiabetes related activities and second section contains 4 questions that focus in attitude of pharmacists toward healthcare professionals and their involvement in diabetes treatment using the third version of the Diabetes Attitude Scale (DAS-3).

Sample: An updated list of community pharmacies in Jordan was taken from the JPA (Jordan pharmacy association), the list contains 1,000 community pharmacies across the country. Five hundred (50\%) community pharmacists were randomly selected.

The survey: The study needs three months (February, March and April, 2011). Pharmacists were informed that the survey was about their experience and attitude toward diabetes and the researcher introduced himself as a researcher from the Faculty of Pharmacy, the Jordan University of Science and Technology.

\section{Results}

\subsection{Activities toward Diabetes Related Activity}

In the present study the survey was distributed to 500 community pharmacists across the country with 422 complete questionnaires (84.4\%). More than half of pharmacists showed practicing pharmacy for five years or less and obtaining their degrees from Jordanian public Universities. The most of pharmacists (55.5\%) showed that less than 20 diabetic patients visited their pharmacy each week and they are sometimes counsel diabetic patients.

When asked about activities related to blood glucose monitoring in diabetes patients, $30.3 \%$ of pharmacists showed that they always counsel patients on the use of a blood glucose meter including how to obtain a blood sample. Pharmacists showed similar activities toward diabetes blood monitoring regarding the appropriate times to check blood glucose (30.8\%) the current treatment targets for blood glucose (33.6\%) and the interpretation of A1C results (26.1\%). However, this was not consistent for counseling patients on identifying the potential causes of abnormal values (17.5\%).

A small part of pharmacists showed that they always counsel patients regarding the handling (18\%), proper use (10\%), and administration (mixing insulin, injection technique, timing of injection, rotation of sites) (6.6\%) of Insulin. Furthermore, a small part of pharmacists showed that they always counsel patients regarding oral anti diabetic.

Pharmacists did not seem to communicate with patients regarding other co-morbidities that may be present along with diabetes. A small part of pharmacists showed that they always counsel patients regarding different diseases that are present with diabetes in most cases. The latter included working with the patient to ensure that they stick to the current recommendations for anti-platelet therapy (11.8\%) that the patient's blood pressure is strictly monitored (18.5\%) that the patient's cholesterol level is under control (6.6\%) and counseling patients about symptoms and implications of depression (3.8\%).

With regards to activities related to life style modification in diabetic patients, a small part of pharmacists showed that they always counsel smoking patients regarding risks of continuing tobacco use (27.7\%). A small part of pharmacists showed that they always counsel patients regarding the importance of immunization for influenza and pneumococcal pneumonia in diabetes $(10.4 \%)$. About one quarter provided basic information on diet as it relates to diabetes management (27.5\%) and basic information on exercise as it relates to diabetes management (28.4\%). Further results are shown in Table 1.

\subsection{Attitude, Views and Experiences regarding Diabetes and Healthcare Professionals}

When asked about their attitudes towards healthcare 
Table 1 Activities toward diabetes related activity.

\begin{tabular}{|c|c|c|c|c|}
\hline & Never & Rarely & Often & always \\
\hline \multirow{2}{*}{$\begin{array}{l}\text { Counsel on the use of a blood glucose meter including how to obtain a } \\
\text { blood sample }\end{array}$} & 66 & 116 & 112 & 128 \\
\hline & $15.6 \%$ & $27.5 \%$ & $26.5 \%$ & $30.3 \%$ \\
\hline \multirow{2}{*}{ Counsel on the appropriate times to check blood glucose } & 66 & 128 & 100 & 128 \\
\hline & $15.6 \%$ & $30.3 \%$ & $23.7 \%$ & $30.8 \%$ \\
\hline \multirow{2}{*}{ Counsel on the current treatment targets for blood glucose } & 60 & 88 & 132 & 142 \\
\hline & $14.2 \%$ & $20.9 \%$ & $31.3 \%$ & $33.6 \%$ \\
\hline \multirow{2}{*}{ Counsel on the interpretation of $\mathrm{A} 1 \mathrm{C}$ results } & 92 & 138 & 82 & 110 \\
\hline & $21.8 \%$ & $32.7 \%$ & $19.4 \%$ & $26.1 \%$ \\
\hline \multirow{2}{*}{ Identify potential causes of abnormal values } & 72 & 178 & 98 & 74 \\
\hline & $17.1 \%$ & $42.2 \%$ & $23.2 \%$ & $17.5 \%$ \\
\hline \multirow{2}{*}{ Counsel on the appropriate handling and storage of insulin } & 100 & 138 & 108 & 76 \\
\hline & $23.7 \%$ & $32.7 \%$ & $25.6 \%$ & $18 \%$ \\
\hline \multirow{2}{*}{ Counsel on the appropriate use of syringes and needles } & 162 & 126 & 92 & 42 \\
\hline & $38.4 \%$ & $29.9 \%$ & $2.8 \%$ & $10 \%$ \\
\hline \multirow{2}{*}{$\begin{array}{l}\text { Counsel on appropriate insulin administration (mixing insulin, injection } \\
\text { technique, timing of injection, rotation of sites) }\end{array}$} & 144 & 158 & 92 & 28 \\
\hline & $34.1 \%$ & $37.4 \%$ & $21.8 \%$ & $6.6 \%$ \\
\hline \multirow{2}{*}{ Describe potential adverse effects of each oral antidiabetic drug } & 94 & 134 & 148 & 46 \\
\hline & $22.3 \%$ & $31.8 \%$ & $35.1 \%$ & $10.9 \%$ \\
\hline \multirow{2}{*}{ Counsel on current recommendations for anti-platelet therapy } & 92 & 156 & 124 & 50 \\
\hline & $21.8 \%$ & $37 \%$ & $29.4 \%$ & $11.8 \%$ \\
\hline \multirow{2}{*}{$\begin{array}{l}\text { Provide education on the importance of controlling blood pressure in } \\
\text { diabetes }\end{array}$} & 84 & 144 & 112 & 78 \\
\hline & $19.9 \%$ & $34.1 \%$ & $26.5 \%$ & $18.5 \%$ \\
\hline \multirow{2}{*}{ Counsel on the current treatment targets for cholesterol in diabetes } & 160 & 150 & 84 & 28 \\
\hline & $37.9 \%$ & $35.5 \%$ & $19.9 \%$ & $6.6 \%$ \\
\hline \multirow{2}{*}{ Provide education on risks of continued tobacco use to smokers } & 52 & 124 & 130 & 116 \\
\hline & $12.3 \%$ & $29.4 \%$ & $30.8 \%$ & $27.5 \%$ \\
\hline \multirow{2}{*}{$\begin{array}{l}\text { Provide education about the importance of immunization for influenza and } \\
\text { pneumococcal pneumonia in diabetes }\end{array}$} & 108 & 168 & 102 & 44 \\
\hline & $25.6 \%$ & $39.8 \%$ & $24.2 \%$ & $10.4 \%$ \\
\hline \multirow{2}{*}{ Provide basic information on diet as it relates to diabetes management } & 54 & 76 & 176 & 116 \\
\hline & $12.8 \%$ & $18 \%$ & $41.7 \%$ & $27.5 \%$ \\
\hline \multirow{2}{*}{ Provide basic information on exercise as it relates to diabetes management } & 74 & 68 & 160 & 120 \\
\hline & $17.5 \%$ & $16 \%$ & $37.9 \%$ & $28.4 \%$ \\
\hline \multirow{2}{*}{ Review the patient's drug profile to identify drugs that are renally cleared } & 170 & 164 & 74 & 14 \\
\hline & $40.3 \%$ & $38.9 \%$ & $17.5 \%$ & $3.3 \%$ \\
\hline \multirow{2}{*}{ Counsel on the symptoms of neuropathic pain } & 108 & 132 & 140 & 42 \\
\hline & $25.6 \%$ & $31.3 \%$ & $33.2 \%$ & $10 \%$ \\
\hline \multirow{2}{*}{ Provide education on the risk of erectile dysfunction in diabetes } & 158 & 136 & 84 & 44 \\
\hline & $37.4 \%$ & $32.2 \%$ & $19.9 \%$ & $10.4 \%$ \\
\hline \multirow{2}{*}{ Counsel on good foot care techniques. } & 92 & 156 & 122 & 52 \\
\hline & 21.85 & $37 \%$ & 28.95 & $12.3 \%$ \\
\hline
\end{tabular}

professionals (including pharmacists) involvement in diabetes care and treatment, the most of pharmacists thought that healthcare professionals should be trained to communicate well with their patients (70.1\%), health care professionals should be taught how daily diabetes care affects patients' lives (55.9\%), that it is important for the health care professionals to learn counseling skills (55.9\%) and healthcare professional educational campaigns are important to prevent diabetes (72\%).

Further results are shown in Table 2 and Fig. 2.

\section{Discussion}

This survey evaluates the pharmacists' experiences and knowledge toward diabetes management.

Almost two third of the pharmacists in this study showed that they always or often counsel diabetic patient on the current treatment targets for blood glucose, the interpretation of $\mathrm{A} 1 \mathrm{C}$ results and the use of a blood glucose meter including how to obtain a blood sample. This consistence with a survey that took part in Canada and Norway. The latter studies showed that 
Table 2 Attitude, views and experiences regarding diabetes and healthcare professionals.

\begin{tabular}{llllll}
\hline & Strongly agree & agree & Neutral & disagree & Strongly disagree \\
\hline Health care professionals who treat people with diabetes & 296 & 94 & 14 & 8 & 10 \\
should be trained to communicate well with their patients & $70.1 \%$ & $22.3 \%$ & $3.3 \%$ & $1.9 \%$ & $2.4 \%$ \\
Health care professionals should be taught how daily & 236 & 158 & 14 & 10 & 4 \\
diabetes care affects patients' lives & $55.9 \%$ & $37.4 \%$ & $3.3 \%$ & $2.4 \%$ & $.9 \%$ \\
It is important for the health care professionals who teach & 236 & 146 & 16 & 8 & 16 \\
people with diabetes to learn counseling skills & $55.9 \%$ & $34.6 \%$ & $3.8 \%$ & $1.9 \%$ & $3.8 \%$ \\
Healthcare professional educational campaigns are & 304 & 98 & 12 & 4 & 4 \\
important to prevent DM & $72 \%$ & $23.2 \%$ & $2.8 \%$ & $.9 \%$ & $.9 \%$ \\
\hline
\end{tabular}

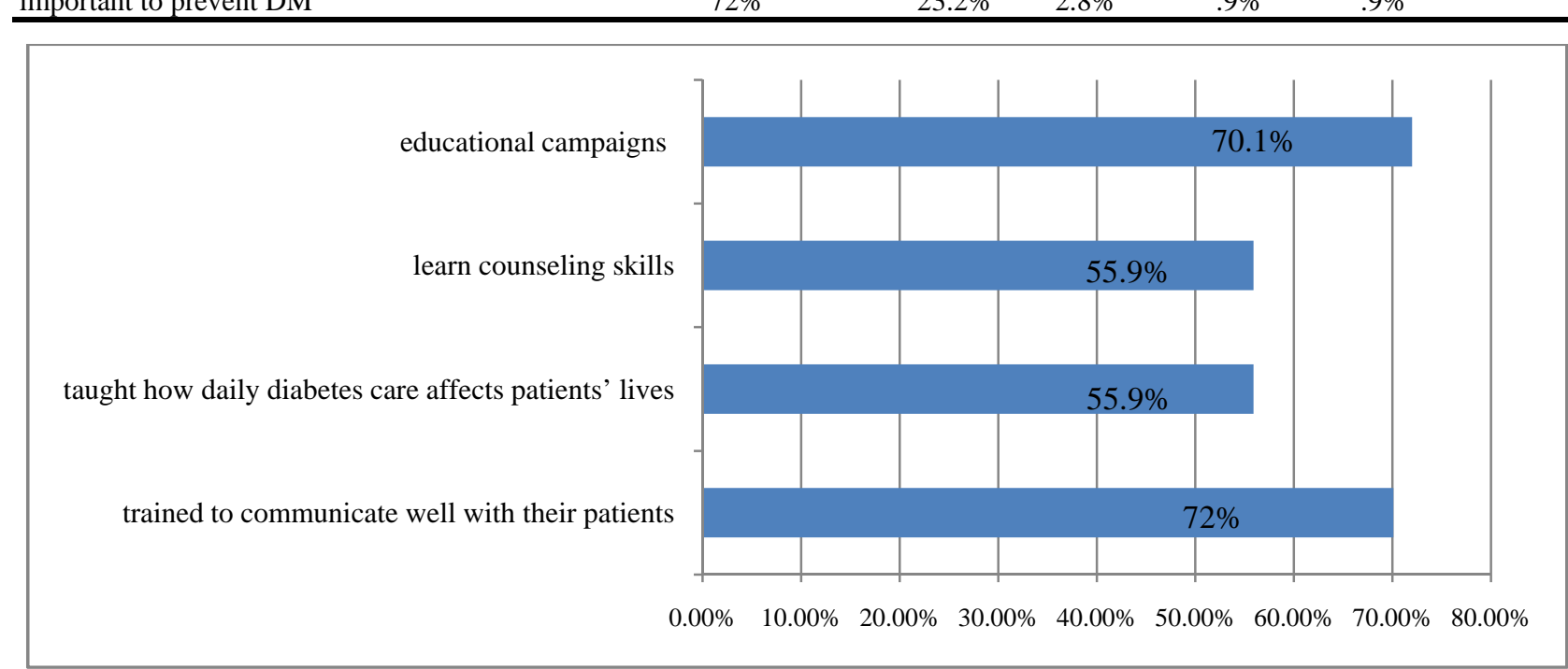

Fig. 1 Attitude regarding healthcare professionals.

almost approximately two-thirds of pharmacists claimed that they regularly provide education to patients on the use of blood glucose meters, appropriate times to check blood glucose and current treatment goals $[11,12]$.

Not counseling diabetic patients in risk of smoking, alcohol abuse and benefit of exercise and diet control showed in the present study was found to be consistent with previous studies that took part in the North East of England and India to measure the community pharmacist's role in the management of diabetes [13, 14]. Heaton and Frede mentioned that the physicians were not able to provide sufficient counselling to diabetic patients regarding lifestyle behaviour while the pharmacist was found to carry out this task effectively [15]. However, according to Kotecki et al., it was showed that the main barriers of effective patient counseling were working constrains, physical design of the pharmacy and lack of knowledge [14]. These barriers may apply to Jordanian community pharmacist as well, leading to the alarming lack of pharmacist-patient interaction.

This study shows that a small part of pharmacists counsel diabetic patients about signs, symptoms, frequency, and causes of hypoglycaemia. Similar results have been shown by a survey that took part in Canada in which a small part of pharmacist showed that they counsel diabetic patients about hypoglycaemic symptoms, signs, frequency and causes of hypoglycaemia [11]. This deficiency of diabetic patient counselling may lead to a lack of awareness of hypoglycaemic and hyperglycemic symptoms especially in elderly. This has been seen in a study that survived 57 elderly living with Type 2 diabetes in the United Kingdom. This study showed that $18 \%$ did not know what action to take with hypoglycemia and $46 \%$ did not know any hyperglycemic symptoms or signs [16]. 
The pharmacists of this study showed that the primary obstacles to providing more diabetic services are time constrain (20\%) and lack of continuous education program (71\%). These results are consistent with a previously published study that survived 233 community pharmacists. The latter study indicated the common barriers to the pharmacists' communication with patients were the pharmacists being busy (58\%) the pharmacists' low level of knowledge (33\%) [17]. So, as indicated in this study, the lack of time is the most significant barrier against the pharmacist's role in patient counseling [18-20].

Further cooperation between the Ministry of Health, the Pharmaceutical and Medical Associations and Jordanian Universities is essential to provide effective educational programs towards good pharmacists' skills in disease management. This will enhance the current community pharmacy services [21].

\section{Conclusion}

Pharmacists should be provided with continuous diabetes education programs to increase and enhance their counseling skills. This is supported by the results of a previous study that took part in Canada which found that pharmacists with CDE (continues diabetes education) programs perform counseling to diabetic patients more than those without certified.

\section{References}

[1] Ajlouni, K., Jaddou, H., and Batieha, A. 1998. "Diabetes and Impaired Glucose Tolerance in Jordan: Prevalence and Associated Risk Factors.” Intern. Med. 244: 317-23.

[2] Shereen Arent, J. D. 2002. "The Role of Health Care Professionals in Diabetes Discrimination Issues at Work and School.” Diabetes Spectrum 15: 217-21.

[3] Mühlhauser, J., and Kasper, G. M. 2006 “Understanding of Diabetes Prevention Studies: Questionnaire Survey of Professionals in Diabetes Car.” Diabetologia 49: 1742-6.

[4] Campbell, R. K. 2002. "Role of the Pharmacist in Diabetes Management.” Am. J. Health Syst. Pharm. 59: S18-S21.

[5] Hawkins, D., Bradberry, J. C. Cziraky, M. J., Talbert, R. L., Bartels, D. W., Cerveny, J. D. and National Pharmacy Cardiovascular Council. 2002. "National Pharmacy
Cardiovascular Council Treatment Guidelines for the Management of Type 2 Diabetes Mellitus: Toward Better Patient Outcomes and New Roles for Pharmacists.” Pharmacotherapy 22: 436-44.

[6] Rodriguez de Bittner, M., and Haines, S. T. 1997. "Pharmacy-Based Diabetes Management: A Practical Approach.” J. Am. Pharm. Assoc. (Wash) NS37: 443-55.

[7] Guirguis, L. M., and Johnson, J. A. 2003. "Diabetes: Are Pharmacists Making an Impact on Patient Outcomes?” Can. Pharm. J. 136: 24-8.

[8] Leal, S., Glover, J. J., Herrier, R. N., and Anthony, F. 2004. "Improving Quality of Care in Diabetes through a Comprehensive Pharmacist-Based Disease Management Program.” Diabetes Care 27: 2983-4.

[9] Blenkinsopp, A., and Hassey, A. 2005. "Effectiveness and Acceptability of Community Pharmacy-Based Interventions in Type 2 Diabetes: A Critical Review of Intervention Design, Pharmacist and Patient Perspectives.” Int. J. Pharm. Pract. 13: 231-40.

[10] Wubben, D. P., and Vivian, E. M. 2008. "Effects of Pharmacist Outpatient Interventions on Adults with Diabetes Mellitus: A Systematic Review.” Pharmacotherapy 28: 421-36.

[11] Simpson, S. H., Haggarty, S., Johnson, J. A., Schindel, T. J., Tsuyuki, R. T., and Lewanczuk R. 2009. "Survey of Pharmacist Activities and Attitudes in Diabetes Management.” Canadian Pharmacists Journal 142: 128-34.

[12] Kjome, R. L., Sandberg, S., and Granås, A. G. 2008. "Diabetes Care in Norwegian Pharmacies: A Descriptive Study.” Pharm. World Sci. 30: 191-8.

[13] Abduelkarem, A. R., Sackville, M. A., Morgan, R. M., Sackville, M. P., and Hildreth, A. J. 2003. "Views and Practices of Community Pharmacists regarding Services for People with Type 2 Diabetes.” Int. J. Pharm. Pract. 11: 161-8.

[14] Kotecki, J. E., Elanjian, S. I., and Torabi, M. R. 2000. "Health Promotion Beliefs and Practices among Pharmacists.” J. Am. Pharm. Assoc. 40: 773-9.

[15] Heaton, P. C. 2006. "Patients' Need for More Counseling on Diet, Exercise, and Smoking Cessation: Results from the National Ambulatory Medical Care Survey.” J. Am. Pharm. Assoc. 46: 364-9.

[16] Pegg, A., Fitzgerald, F., Wise, D., Singh, B. M., and Wise, P. H. 1991. "A Community-Based Study of Diabetes-Related Skills and Knowledge in Elderly People with Insulin-Requiring Diabetes.” Diabet Med. 8: 778-81.

[17] Otero, L. M., Zanetti, M. L., and Ogrizio, M. D. 2008. "Knowledge of Diabetic Patients about Their Disease before and after Implementing a Diabetes Education Program.” Rev. Latino-Am Enfermagem 16: 231-7. 
[18] McKenney, J. M. 1993. "An Evaluation of Cholesterol Screening in Community Pharmacies.” Am. Pharm. NS33: 34-40.

[19] Crawford, N. 1992. "The Pharmacists Office Centre: Providing Quality Care.” Am. Pharm. NS32: 36-8.

[20] Nykamp, D., and Barnett, C. W. 1992. "Use of Stationary
Automated Blood Pressure Devices in Pharmacies.” Am. Pharm. NS32: 33-6.

[21] Bell, J. S., Väänänen, M., Ovaskainen, H., Na“rhi, U., and Airaksinen, M. S. 2007. "Providing Patient Care in Community Pharmacies: Practice and Research in Finland.” Ann Pharmacotherapy 41: 1039-46. 